\title{
LA MUJER EN EL COMERCIO INFORMAL EN LA CIUDAD DE HUANCAYO
}

\section{THE WOMAN IN THE INFORMAL TRADE IN THE CITY HUANCAYO}

\author{
Filoter Tello Yance'
}

\begin{abstract}
RESUMEN
Actualmente en la ciudad de Huancayo se viene incrementando la participación de la mujer en el comercio informal. Este fenómeno social ha originado tugurios en las calles de mayor movimiento comercial. Del $100 \%$ de comerciantes informales, el $90 \%$ de comerciantes son mujeres y sólo el $10 \%$ varones. Una de las causas del comercio informal es la pobreza, falta de empleo, los bajos ingresos y la sobrecarga tributaria, que no les permite formalizarse y acceder a un establecimiento fijo con las condiciones adecuadas en lugar apropiado La inserción de la mujer en este sector es para aportar a la economía familiar cuando el ingreso del jefe de familia es insuficiente, o para mantener a la familia cuando es el único sostén. El ingreso que obtienen es menos del salario mínimo vital. Estas mujeres trabajan en las calles en condiciones infrahumanas a falta de un lugar adecuado. Cuentan generalmente con un capital de 50 a 350 nuevos soles, es una ocupación sólo para la subsistencia familiar. La mayoría de las mujeres ambulantes tienen una carga de deudas .Ninguna institución se interesa por el bienestar de este grupo social. Trabajan en condiciones de exclusión y marginación social. Carecen de apoyo crediticio que otorgan las entidades financieras El nivel de educación de estas mujeres es de primaria y de secundaria completo. Los problemas de las mujeres informales están asociados a la, ausencia de un lugar adecuado, competencia entre las vendedoras, bajos ingresos, abuso de los policías municipales, las trabas fiscales y administrativas.
\end{abstract}

Palabras clave: participación de la mujer, comercio informal, desempleo y pobreza

\begin{abstract}
At the moment in the city of Huancayo one comes increasing the woman's participation in the informal trade. This social phenomenon has originated dives in the streets of more commercial movement. Of $100 \%$ of informal merchants, $90 \%$ of merchants is women and only $10 \%$ males. One of the causes of the informal trade is the poverty, it lacks of employment, the low revenues and the tributary overload that it doesn't allow them to be formalized and to consent to a fixed establishment with the conditions adapted in appropriate place the woman's insert in this sector it is to contribute to the family economy when the family boss's entrance is insufficient, or to maintain to the family when it is the only support. The entrance that you/they obtain is less than the vital minimum wage. These women work in the streets under conditions infra human for lack of an appropriate place. They generally have a capital from 50 to 350 "Nuevos Soles", it is only an occupation for the family subsistence. Most of the travelling women have a load of debts. No institution is interested in the well-being of this social group. They work in exclusion conditions and social imagination. They lack credit support that you/they grant the financial entities The level of these women's education it is of primary and of secondary I complete. The problems of the informal women are associated to the, absence of an appropriate place, competition among the salespersons, low revenues, the municipal policemen's abuse, the fiscal and administrative obstacles.
\end{abstract}

Key words: The woman's participation, informal trade, unemployment and poverty 


\section{INTRODUCCIÓN}

El comercio informal en la ciudad de Huancayo, está articulado como consecuencia de la migración del campo a la ciudad. Las causas que motivaron las migraciones tanto internas como hacia el exterior es de carácter multivariable y multicausal, es decir, migran por motivos económicos (busca de trabajo y de ingreso, bajos ingresos económicos, desempleo, subempleo, etc.); sociales (asuntos familiares, salud, educación, etc.); políticos (violencia política); desastres naturales (huaycos, movimientos sísmicos, etc.).

Las personas que llegan del campo a la ciudad, generalmente son de mano de obra barata y no calificada por lo que tienen dificultades para acceder a ocupaciones calificadas; sin embargo tienen necesidades de alimentación, vivienda, vestido, educación, salud, recreación, y seguridad, además no existe fuente de ocupación , el mercado laboral está saturado y muchos jóvenes con título profesional forman parte de la población económicamente no ocupada, este hecho en nuestro Perú es un problema general y ante esta situación tan dramática el comercio informal constituye el fuente de trabajo y de ingreso, denominado por muchos como el autoempleo.

En la ciudad de Huancayo según los datos recogidos directamente suman 1673 mujeres en el comercio informal, de los cuales el $10 \%$ (163) son varones quienes contribuyen con el ingreso familiar para alimentar, educar, vestir y velar por la salud de sus hijos y de la familia tanto nuclear y porque no decirlo de la familia extensa (suegro, suegra, yerno, nueras y otros). Indudablemente la investigación se orienta a dar cuenta de la problemática del comercio informal, no trata sólo caracterizar las causas, las consecuencias, sino también proponer soluciones viables mediante una propuesta técnica para ser implementada mediante una decisión política.

Actualmente la Municipalidad Provincial de Huancayo como ente regulador establece normas de ordenamiento, de ubicación y de organización del comercio tanto formal e informal; sin embargo existe conflictos entre vendedores ambulantes y el Municipio, los vendedores formales con informales y en este proceso se evidencia en el mal trato a la mujer por parte de los policías municipales, son víctimas de robos, maltratos, de exclusión y de marginación sociales. Trabajan en condiciones infrahumanas en la intemperie, tragando el polvo todos los días, en barro, agua, lluvia y la insolación. Por eso, estas mujeres ambulantes, conocidas como "placeras" demuestran una conducta agresiva como reflejo de la realidad social en la cuales se desempeñan.

\section{MATERIAL Y MÉTODOS}

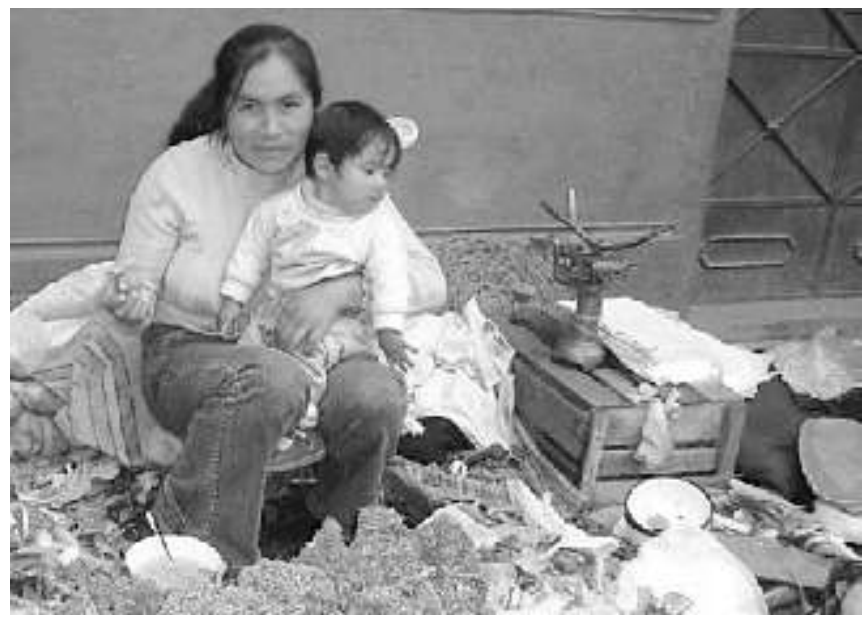

Los materiales utilizados en esta investigación fueron los padrones de los ambulantes por calles y por secciones elaborados por la Municipalidad de Huancayo dentro de su jurisdicción territorial sin incluir a los distritos de El Tambo y Chilca. Los cuestionarios como instrumentos de recopilación de la información necesaria.

En el proceso de la investigación se utilizaron diversos métodos: la cuantitativa para explicar el ingreso y otros, también la cualitativa como los testimonios para conocer la procedencia y las causas de la migración y la inserción al comercio informal. Las técnicas usadas fueron la observación directa y el cual ha permitido identificar por calles el número de giro de negocios existentes y las condiciones en las cuales trabajan, se pudo observa igualmente los enfrentamientos diarios que se suscitan entre los ambulantes y los policías municipales, éstos últimos les quita la mercadería a las mujeres y no les devuelve, poniendo en riesgo la estabilidad financiera y a la postre conduce a la quiebra económica

Tabla 1. Principales problemas del comercio ambulatorio.

\begin{tabular}{lcc}
\hline Problemas & $\mathbf{N}^{\circ}$ & $\%$ \\
\hline 1. Falta de capital & 7 & 6 \\
2. Falta de lugar adecuado & 3 & 2 \\
3. Competencia & 4 & 3 \\
4. Bajos ingresos & 5 & 4 \\
5. Abuso de los policias municipales & 9 & 6 \\
6. Falta de seguridad (robos) & 2 & 1 \\
7. Falta de capital y seguridad & 3 & 2 \\
8. Falta de capital y lugar adecuado & 21 & 15 \\
9. Falta de capital y competencia & 14 & 10 \\
10. Falta de capital y bajos ingresos & 6 & 4 \\
11. Falta de capital y abuso de policias & 4 & 3 \\
12. Falta de lugar adecuado y competencia & 10 & 7 \\
13. Falta de lugar adecuado y seguridad & 16 & 11 \\
14. Competencia y falta de seguridad & 10 & 7 \\
15. Bajos ingresos, baja venta y competencia & 16 & 11 \\
16. Bajos ingresos y falta de seguridad & 11 & 8 \\
\hline Total & $\mathbf{1 4 0}$ & $\mathbf{1 0 0}$ \\
\hline
\end{tabular}

Elaboración propia. 


\section{RESULTADOS}

En la Tabla 1, se indica que el $15 \%$ de las mujeres que trabajan en el comercio informal de la ciudad de Huancayo, opinan que la falta de capital y un lugar adecuado es el principal problema del comercio ambulatorio; el $11 \%$ consideran, la falta de lugar adecuado, seguridad, bajos ingreso, baja venta y la competencia que existen entre las vendedoras ambulantes y éstas con los comerciantes formales

Tabla 2. "El trabajo es sacrificado en el comercio ambulatorio",

\begin{tabular}{crc}
\hline Categorias & $\mathbf{N}^{\circ}$ & $\%$ \\
\hline Si & 118 & 84 \\
No & 22 & 16 \\
Total & 140 & 100 \\
\hline
\end{tabular}

Fuente: Elaboración propia.

serían los otros problemas existentes en el comercio ambulatorio de Huancayo; el $10 \%$ de las mujeres afirman que el problema es la falta de capital y competencias, asimismo los bajos ingresos y la falta de seguridad, serían el problema, pero considerado de menor importancia, igualmente el abuso que cometen los policías municipales es el otro problemas que afrontan las vendedoras.

A todos estos problemas es necesario incluir los ruidos que originan el parque automotor, la bocina de los triciclos, la contaminación ambiental, la lluvia, barro, el frío, calor solar, las peleas entre las trabajadoras por quitar el lugar de venta o "puesto" en la calle. En conclusión la tabla 1, nos muestra que el comercio informal existente en la ciudad de Huancayo de carácter multicausal y la solución debe responder a esta naturaleza tan compleja como parte de la política social del Estado y de la gestión de los gobiernos locales de la provincia de Huancayo y del país.

Según la tabla 2 , el $84 \%$ de las vendedoras ambulantes consideran que el comercio informal es muy sacrificado y esta idea es reflejo de los múltiples problemas que afronta de manera estoica y significa soportar con tolerancia muchos retos y adversidades. Primero estar desempleada y sin ingreso, con una carga de necesidades de alimentación, vestido, vivienda, educación, salud, pago de servicios de transporte, recreación y otros compromisos; pone a la persona en una vida con existencia desesperante y optar a convertirse en vendedora ambulante, significa generar el autoempleo como estrategia de sobrevivencia y asumir todas las consecuencias sociales que supone este tipo de trabajo. Socialmente las vendedoras ambulantes son marginadas y reprimidas por la policía municipal, son víctimas de muchos abusos de los derechos humanos y ante esta situación ninguna institución de los derechos humanos asume la defensa.

Es sacrificado por muchas razones: primero no tienen dinero para iniciar el negocio y los usureros (personas que prestan dinero de manera independiente de las instituciones bancarias cobran con altas tasas de interés) se aprovechan para otorgarle el préstamos a cambio de una prenda y con $20 \%$ de interés mensual, significa cien soles gana el interés de 20 soles cada mes, no tienen un

Tabla 3. Número de horas que trabaja al día.

\begin{tabular}{lrr}
\hline $\mathbf{N}^{\circ}$ de Horas & $\mathbf{N}^{\circ}$ & $\%$ \\
\hline 2 & 1 & 0,7 \\
4 & 3 & 2,1 \\
5 & 1 & 0,7 \\
6 & 8 & 5,7 \\
7 & 3 & 2,1 \\
8 & 15 & 10,7 \\
9 & 6 & 4,3 \\
10 & 22 & 15,7 \\
11 & 10 & 7,1 \\
12 & 34 & 24,3 \\
13 & 15 & 10,7 \\
14 & 6 & 4,3 \\
No responde & 16 & 11,4 \\
Total & $\mathbf{1 4 0}$ & $\mathbf{1 0 0 , 0}$ \\
\hline
\end{tabular}

Fuente: Elaboración propia.

puesto fijo donde expender humanamente sus productos, son perseguidas por la policía

Tabla 4. "Cuentan con seguro de vida".

\begin{tabular}{lrr}
\hline Seguro de salud & $\mathbf{N}^{\circ}$ & $\%$ \\
\hline Si & 12 & 9 \\
No & 128 & 91 \\
Total & $\mathbf{1 4 0}$ & $\mathbf{1 0 0}$ \\
\hline
\end{tabular}

Fuente: Elaboración propia.

municipal, quienes les despojan de sus pertenencias, exigen coimas en dinero o en especia y los acosa a las más jóvenes para dejarlas vender. Trabajan en permanente riesgo de los accidentes de tránsito, robos, la lluvia, barro, frío, polvareda, son víctimas de insultos, agresiones y de los maltratos físicos. Lo cual significa la violencia contra la mujer y los agresores son muchos, iniciando de la policía 
municipal, los comerciantes formales que votar de la puerta de sus establecimientos por considerarlas competidoras desleales, peleas entre las vendedoras por disputar un lugar en la calle, dejan sus hijos (as) abandonadas en sus domicilios por la necesidad de llevar un pan a sus hogares producto de su sacrificio

En la Tabla 3, se observa que el $24 \%$ de las mujeres trabajan 12 horas diarias; el 15,7\% 10 horas diarias y $10,7 \%$ más de 13 horas diarias. El número de horas/ hombre al día está en función al giro de negocio. Las mujeres que expenden comida trabajan entre 4 y 5 horas, luego están en otras actividades también lucrativas en cambio las que venden ropa hecha, abarrotes, frutas, verduras trabajan entre 12 y 13 horas diarias.

Tabla 5. "¿Qué días descansan?"

\begin{tabular}{lrr}
\hline Dias de descanso & $\mathbf{N}^{\circ}$ & $\%$ \\
\hline Domingo & 3 & 2 \\
Lunes & 25 & 18 \\
Martes & 15 & 11 \\
Miércoles & 3 & 2 \\
Jueves & 4 & 3 \\
Sábado & 2 & 1 \\
Total & 52 & 37 \\
No descansa & 88 & 63 \\
Total & $\mathbf{1 4 0}$ & $\mathbf{1 0 0}$ \\
\hline
\end{tabular}

Fuente: Elaboración propia.

De acuerdo la tabla 4, el $91 \%$ de las mujeres carecen de los servicios de seguro de salud, la vida de estas mujeres está en permanente riesgo.

La tabla 5, nos indica que el $63 \%$ no toma sus día libres para descansar, lo cual nos indica que es el único sustento del hogar; el $18 \%$ de las mujeres ambulantes descansa los días lunes, el 11 \% el día martes y las demás cualquier día de la semana, y está en función a las prioridades de los compromisos y quehaceres domésticas a cumplir, tales como las actividades reproductivas (realizar la limpieza de la casa o para cumplir actividades sociales). Los días sábados y domingos como es de mayor venta, todos los empleados públicos y trabajadores del sector privado acuden al mercado a realizar sus compras, razón por la cual priorizan no descansar, sólo algunas después de haber aprovechado la venta en la mañana prefieren salir en la tarde para asistir a los matrimonios del día sábado que es una costumbre en el valle del Mantaro.

Según la tabla 6, el motivo principal para trabajar en el comercio informal es la carencia de fuentes de empleo y el $21 \%$ sirve como actividad complementaria al salario del esposo que gana muy poco.

Tabla 6. Motivos para trabajar en el comercio informal.

\begin{tabular}{lrr}
\hline Motivos & $\mathbf{N}^{\circ}$ & $\frac{9}{8}$ \\
\hline 1. No hay empleo & 70 & 50 \\
2. Sirve de complemento al salario del esposo & 30 & 21 \\
3. Es madre soltera y necesita mantener a sus hijos & 20 & 14 \\
4. Falta de capital y lugar adecuado & 6 & 4 \\
5. No paga impuestos & 10 & 7 \\
6. Es costoso la apertura de un negocio formal & 4 & 3 \\
Total & $\mathbf{1 4 0}$ & $\mathbf{1 0 0}$ \\
\hline
\end{tabular}

Fuente: Elaboración propia.

\section{DISCUSIÓN}

En la ciudad de Huancayo, se evidencia la presencia de la mujer en el comercio informal, actividad que funciona al margen de la ley y forma parte de la problemática social, política y económica de la región central. Hasta la fecha los gobiernos locales de turno demostraron incapacidad en la solución definitiva de este fenómeno social. Los ambulantes se ubican en las calles de mayor movimiento comercial y de preferencia en el entorno de los mercados de Raez Patiño, mercado mayorista $N^{\circ} 1$ (ex Maltería Lima) y del Mercado Modelo. Al respecto no existe un consenso claro en la definición de informalidad. Sin embargo, en términos generales éstas comprenden actividades ilícitas como la fabricación y el tráfico de drogas; la comercialización de artículos robados, y actividades de contrabando; así como también actividades que no tienen un carácter estrictamente lícito y que escapan a registro y/o a la regulación del gobierno.

Huancayo, es receptora de emigrantes de las regiones de Ayacucho, Cerro de Pasco, Huanuco, Huancavelica, Apúrimac y de las distintas provincias, distritos y comunidades campesinas de la región Junín, quienes inducieron al acelerando proceso de urbanización, a la formación de poblaciones urbano- marginales, desde esto lugares asisten todos los días a cumplir con sus actividades cotidianas y trabajan en permanente conflictos con los policías municipales.

Hernando De Soto en su libro "El otro sendero" sostiene, "que a medida que la ciudad fue llenándose de gente y su espacio urbano se fue informalizando, otras actividades económicas comenzaron a sufrir una evolución equivalente. Una de ellas fue el comercio, que empezó a ser realizado masivamente al margen y hasta en contra de las normas estatales nominalmente encargadas de regularlo. Surgió, así, el comercio informal que, en 
lo esencial, se desarrolla en las calles- bajo la denominación común de comercio ambulatorio- y en mercados construidos específicamente para salir de las mismas",(1).

"En el caso del comercio ambulatorio, la gente comenzó a invadir la vía pública, cuyo uso es de todos, para realizar sobre ella actos de disposición y utilizarla para su operación comercial sin tener licencias, dar facturas ni pagar impuestos, aunque en algunos casos ha estado favorecida por un régimen de excepción legal que, contra el pago de un derecho llamado "sisa", le hizo gozar de la tolerancia municipal. Al invadir, el ambulante por lo general actúa individualmente. Además, no lo hace en un solo momento, sino de a pocos, como tratando de confirmar sus propios cálculos y la posibilidad de que ocurran consecuencias imprevistas" (ídem).

"La economía formal y el aparato productivo de bienes y servicios han sido incapaces de proporcionar empleo estable y bien remunerado a los trabajadores en general y a los jóvenes y mujeres que se incorporan cada año al mercado de trabajo, lo cual se agudiza por los programas de reajuste económico, las migraciones internas y externas, sobre todo a la gran ciudad, provenientes del campo o de las ciudades pequeñas y marginales, así como por la aplicación indiscriminada de las nuevas tecnologías del aparato productivo". El estructural. Aunque la economía informal inició por la insuficiencia del desarrollo industrial, se ha convertido en una situación permanente, como consecuencia de la crisis del capitalismo. Las políticas neoliberales."La economía informal se ha desarrollado y se consolida como parte de la nueva economía globalizada con el máximo de descentralización operativa". Para los trabajadores de la economía informal ésta actividad ha sido la vía para satisfacer sus necesidades elementales. Entre las que se encuentran las siguientes: Los ingresos que perciben son superiores a los mínimos establecidos que se pagan en el país. La continúa amenaza del desempleo y la inestabilidad en el trabajo asalariado. La idea de independencia frente al trato de los patrones. La ilusión de la mejoría por el esfuerzo propio" (ídem) resumido y reestructurado por Pablo Lasso "Soy una madre que ha subido a interrumpir tu viaje en este vehículo para llevar un pan para mis hijos. No me des la espalda, ni me mires con desprecio cuando pase a ofrecerte estos caramelos de menta a 20 céntimos". Micaela, una limeña de 32 años, tiene tres hijos, su esposo es obrero de la construcción y durante largas temporadas está sin empleo; vende caramelos en el servicio de transporte, obtiene un promedio de 58 dólares al mes, representa un caso extremo de trabajo informal: trabaja por cuenta propia, ocupación que con algunos matices se repite en diversos países de América Latina, el Caribe y en el mundo.

\section{REFERENCIAS BIBLIOGRÁFICAS}

1. Hernando de Soto El otro sendero, Editorial el Barranco, Lima;1986

2. Franco C. Informales: nuevos rostros en la vieja Lima, CEDEP; 1989.

3. Webb R, Fernández Baca G. "Perú en números 2004: Anuario estadístico". Lima. Editorial Instituto Cuánto; 2004.

E-mail: ftello90@hotmail.com 